\title{
AUMENTO DE PRODUTIVIDADE NOS PRODUTOS ACABADOS EM VERGALHÕES CA60 PELA AUTOMAÇÃO DOS SISTEMAS DE VELOCIDADE*
}

José Hugo de Carvalho Souza' Shayene Pinheiro Marques ${ }^{2}$ Antonio Bittencourt da Silvar

\section{Resumo}

Em busca do aumento de produtividade e estabilidade operacional de trefilados e ampliados no setor de acabamento da siderúrgica, na produção de CA60, telas e treliças, foi observado através da metodologia DMAIC a instabilidade operacional no indicador de eficiência do OEE dos equipamentos devido a oscilações de velocidade manual e limitação de velocidade por problemas técnicos. Após as análises de estabilidade nos equipamentos e constatado a necessidade de melhoria do processo foi realizado estudos por meio da técnica de modelagem de Rede de Petri, representando o sistema e modificando a lógica de automação que analisa o status da máquina e a partir desse momento o próprio equipamento toma a decisão segura de acelerar ou desacelerar até a velocidade especificada de "set point" por material produzido, sinalizando antecipadamente ao operador com alarmes visuais e sonoro de forma que o mesmo tenha ciência do que está acontecendo e possa intervir caso julgue necessário. Após a implementação foi capturado um aumento de $6,62 \%$ de OEE e 5,5\% de produtividade total anual no setor, sem custo de aquisição.

Palavras-chave: Automação; Trefilação; Controle; Velocidade.

\section{PRODUCTIVITY INCREASE IN FINISHED CA60 BAR BY AUTOMATION OF SPEED SYSTEMS}

\section{Abstract}

In search of increased productivity and operational stability of drawing and expanded in steel finishing industry, in the production of CA60, mesh and trusses, was observed through the DMAIC methodology operational instability in the OEE efficiency indicator of the equipment due to fluctuations speed manual and limiting speed due to technical problems. After the stability analysis in the equipment and confirmed the need for process improvement studies was conducted by Petri network modeling technique, representing the system and modifying the automation logic that comparing the momentary status of the machine and from the that moment the equipment itself takes the safe decision to accelerate or decelerate to the specified speed "set point" for the material produced, signaling for the operator with visual and audible alarms so that it has been informed of what is happening and to intervene if deemed necessary. After implementation was captured an increase of $6.62 \%$ OEE and $5.5 \%$ of total annual productivity in the sector, without cost.

Keywords: Automation; Wire drawing; Control; Speed.

1 Engenheiro de Automação, Mestrando em Engenharia de Automação, Engenheiro de Manutenção, Laminação a Frio, Votorantim Siderurgia / Universidade Federal de Itajuba (UNIFEI), Barra Mansa, Rio de Janeiro, Brasil.

2 Graduanda em Engenharia de Computação, Estagiaria de Engenharia de Manutenção, Laminação a Frio, Votorantim Siderurgia / UBM, Barra Mansa, Rio de Janeiro, Brasil.

3 Técnico em Eletrônica, Técnico Especialista de Elétrica, Laminação a Frio, Votorantim Siderurgia, Barra Mansa, Rio de Janeiro, Brasil. 


\section{INTRODUÇÃO}

Devido à necessidade de aumento de produtividade e a instabilidade no setor de acabamento da Votorantim Siderurgia, unidade Barra Mansa, fez-se necessário o entendimento dos indicadores que eram mais impactantes no OEE da planta, constatando-se baixos e instáveis resultados no indicador de eficiência. O projeto consiste em otimização de produção através de aumento e estabilização de velocidade nos laminadores a frio, treliças e tela. O conceito do projeto foi empregado igualmente em cada setor, porém existiam particularidades em cada um deles que serão brevemente descritas e solucionadas com técnicas de processo, automação e elétrica a seguir.

Inicialmente, no setor de laminador a frio foram capturados dados amostrais através do software de carta de controle IBA PDA para medir a performance de produtividade, analisando a velocidade em vários momentos da produção. Os dados foram analisados e observamos que existiam oscilações de performance por horário de produção, experiência do colaborador no momento de troca de bobina, passagem de solda, suaves rampas de aceleração e desaceleração, etc. Para tal solução foi criado uma lógica de automação inteligente no CLP $\$ 7300$ e IHM OP17 em plataforma Protool, a partir de uma receita operacional que detecta cada um dos momentos de gap e automatiza o sistema, estreitando-o e estabilizando-o, além disso os inversores de frequência Masterdrive foram reparametrizados. A interação com o homem é feita através de mensagens na $\mathrm{IHM}$, sinalizadores visuais e sonoros.

No setor de telas, o equipamento possuía restrição técnica quanto ao atingimento da velocidade máxima, devido a queima precoce do servomotor e insegurança operacional nos ajustes no setpoint máximo. O motor de lançamento de arame transversal foi estudado e após a comprovação do mesmo ser subdimensionado para a aplicação e também ser comprovado a inviabilidade financeira da troca do modelo do conjunto motoredutor, atuamos no sistema de refrigeração do mesmo através de conceitos de termodinâmica alterando o tipo de troca térmica de AR para ÁGUA, permitindo assim a durabilidade do mesmo na velocidade máxima. Após a possibilidade de alcance de velocidade máxima do equipamento foi criado uma lógica de automação no CLP S7400 e em uma nova IHM OP277 em plataforma WinccFlexible, onde uma receita operacional foi implementada com um sistema inteligente de velocidade semelhante ao do laminador a frio mencionado anteriormente.

No setor de treliças, a oscilação de velocidade era menor, por se tratar de um processo mais estável, porém foi observada uma oportunidade de aumento da velocidade máxima do equipamento, devido ao superdimensionamento das peças mecânica, elétrica e operacional. Para tal alteração, era necessário alterar a lógica de sincronismo do equipamento de forma que o sistema de cabeçote e expedição de treliça esteja sincronizado com o sistema de abastecimento e loops dos arames do zig-zag e banzos superior e inferior. O desenvolvimento da lógica foi realizado no CLP S7300 e na reparametrização dos inversores de frequência Lenze, que limitavam desnecessariamente o limite de frequência máxima de rotação dos motores do loop. Após tais modificações, mensagens foram desenvolvidas na IHM OP17 em plataforma Protool e sinalizadores visuais foram instalados indicando ao operador o momento em que o sistema está operando em velocidade recomendada por lógica. 
O presente estudo detém como objetivo a estabilização de todos os equipamentos mencionados em velocidade máxima automaticamente, possibilitando ao operador interromper temporizadamente o sistema automático para quaisquer analise do equipamento em marcha reduzida. O resultado esperado é de um aumento de OEE em $6,62 \%$ com custo de aquisição próximo a zero.

Diante do exposto, pretende-se mostrar os resultados obtidos através de novas amostras em carta de controle

\section{MATERIAIS E MÉTODOS}

\subsection{Laminador a frio EVG}

No setor de laminação a frio foram realizadas medições através da carta de controle IBAPDA e analisamos estatisticamente com o Minitab cada etapa do processo afim de calcular o número meta do processo de laminação como um todo:

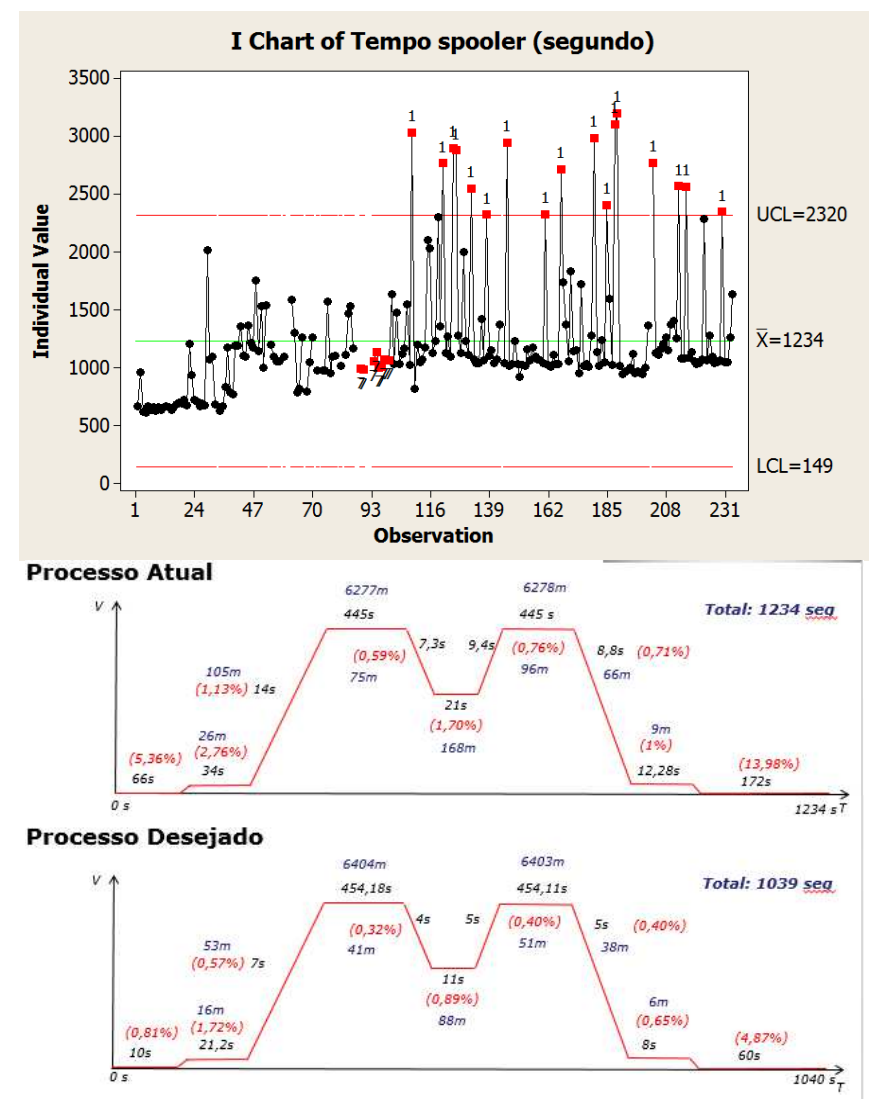

Figura 1: Analise amostral do processo x meta

\subsection{Tela EVG}

No setor de telas, foi utilizado o osciloscópio FLUKE ScopeMeter 199C, realizando analise de espectro do motor de arame de transversal, atual gargalo para atingimento da velocidade máxima do equipamento:

\begin{tabular}{|c|c|c|c|c|c|c|c|c|c|c|c|c|c|c|c|}
\hline Metor type & ${ }_{\ln 1}^{n}$ & $n_{n=1}^{n}$ & 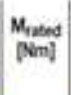 & $\begin{array}{l}P_{\text {Pnatod }} \\
{[\mathrm{WW}]}\end{array}$ & $\frac{v_{\text {rateses }}}{M}$ & $t_{\mid \mathbb{A}}$ & $\underset{[\mathbf{N m}]}{\mathbf{M}_{0}}$ & $\underset{\text { (N) }}{6}$ & $M_{\operatorname{man}}$ & $\begin{array}{l}\operatorname{rothod}_{\text {max }} \\
\left(\min ^{2}\right)\end{array}$ & 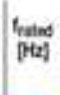 & $\cos 5 \mathrm{~N}$ & 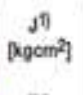 & $\begin{array}{l}m^{11} \\
\text { |xpll }\end{array}$ & 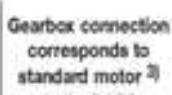 \\
\hline MOFKA $100-22,120$ & & (3510) & 36.0 & (13.2) & $(300)$ & $(28.7)$ & 40.0 & 30.8 & 180 & 8000 & 120 & (6.80) & 72 & 48.2 & 112 , C160 \\
\hline
\end{tabular}

Figura 2: Especificação do servomotor. 

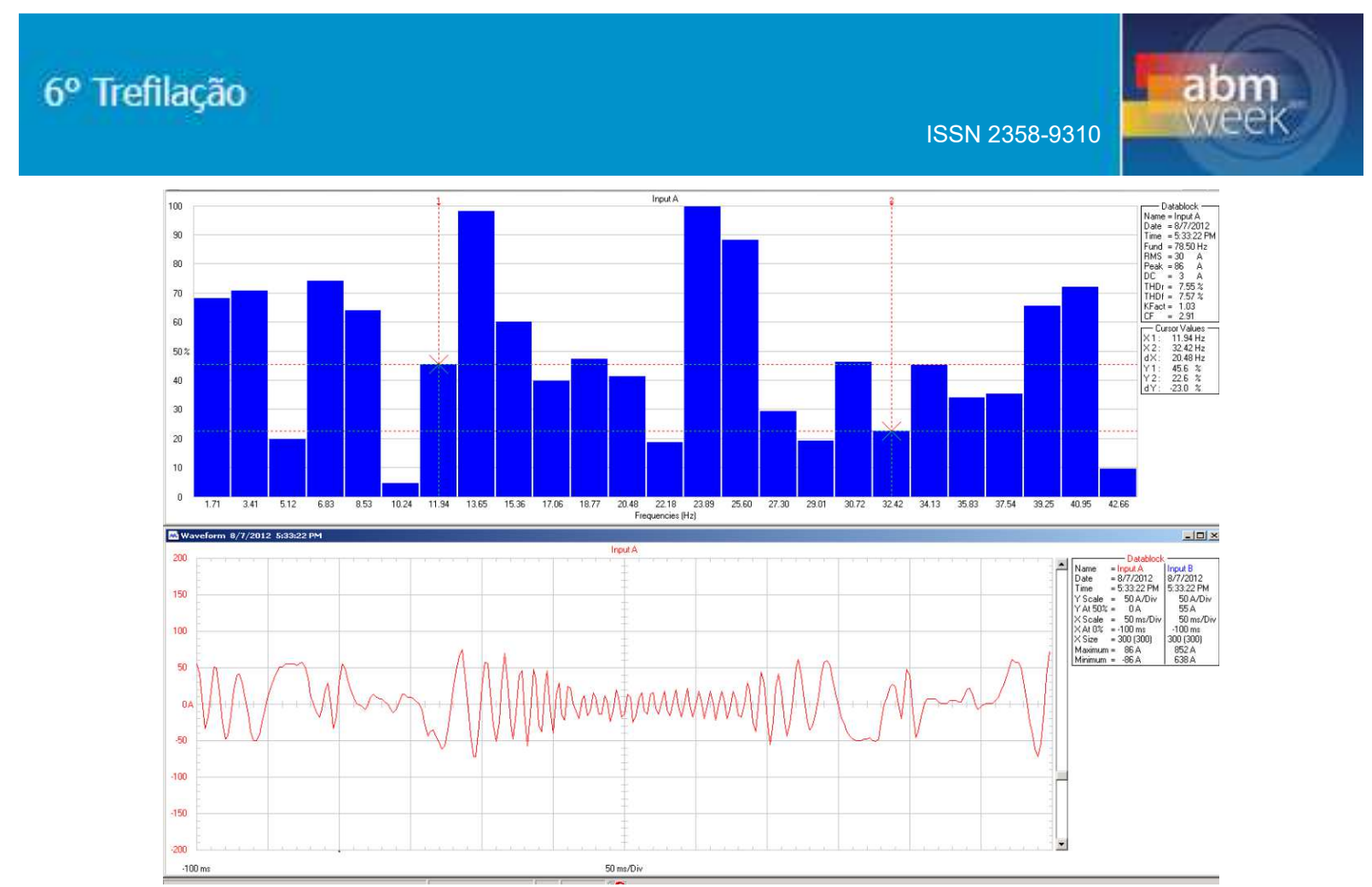

Figura 3: Analise de espectro motor do arame transversal

É possível observar a partir da análise de espectro acima e dados de placa do fabricante que a corrente elétrica nominal do servomotor em velocidade máxima ultrapassa a recomendada pelo fabricante, onde consequentemente existe um aumento térmico interno no enrolamento do motor gerando a queima e curto das bobinas em curto período de tempo por fadiga. Após a conclusão da causa das queimas apresentada acima, foi realizado um estudo de eficácia de troca térmica com o servomotor baseado em conceitos de termodinâmica, onde foi desenvolvido um sistema eficiente de troca térmica com água em serpentinas de cobre que diminuiu a temperatura interna em 15\%, enquadrando-o novamente dentro da classe $\mathrm{H}$ de verniz dos bobinamentos.

Condicional de eficácia: $\quad\left|-k \cdot A \cdot \frac{\Delta T 1}{L} \leq v\right| \rho . C p . \Delta T 2$
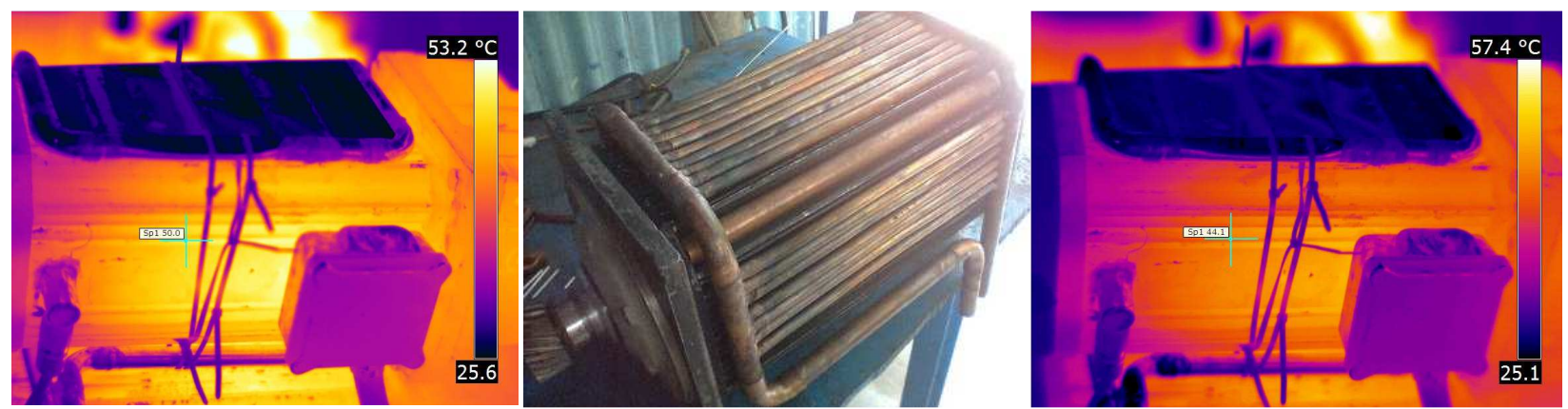

Figura 4: Sistema de refrigeração a agua desenvolvido com serpentinas de cobre e analise termográfica.

Após a comprovação técnica da eficácia da troca térmica e consequentemente a eliminação da restrição de velocidade do equipamento, observou-se a necessidade de se criar um sistema de estabilização de velocidade similar ao do laminador a frio, onde ao selecionar o tipo de tela a ser produzido, o equipamento receberá o "set point'de velocidade e buscará a velocidade automaticamente, sinalizando através de lâmpadas. 


\subsection{Treliça EVG}

No setor de treliças, o projeto comissionado em 2004 limitava a velocidade do equipamento. Motores estratégicos do equipamento foram medidos e analisados com multímetros, amperímetros e dados de placas, além de inversores de frequência, cilindros de solda e rolos operacionais, constatando-se que o equipamento como um todo poderia ser acelerado, desde que ajustes no setor do loop, lógica de sincronismo de automação fossem modificadas e parametrização de inversores de frequência fossem revisados.

\section{RESULTADOS E DISCUSSÃO}

\subsection{Laminador a Frio EVG}

Foi desenvolvido em lógica de automação do CLP S7300 Siemens, um sistema de receita ponteirada em interface com a IHM OP17 em plataforma Protool, afim de diagnosticar o material que está sendo produzido. Além disso, o cálculo de comprimento foi medido através de um encoder incremental, atualizando automaticamente a massa linear do produto limitando assim o peso máximo da bobina de forma que não comprometa o processo seguinte de acabamento. A lógica identifica o momento exato de fabricação do CA60, descobrindo através de sensores se a solda está passando no equipamento e através de encoder se a bobina está no início, meio ou fim. A partir da detecção do momento instantâneo da máquina, um algoritmo inteligente toma a decisão se acelera, freia, estabiliza ou para o equipamento, gerando Alarmes visuais e sonoros sempre que necessário para garantir a segurança das pessoas envolvidas. Após as implementações nesse equipamento, houve um aumento de performance de OEE de 6,67\%.

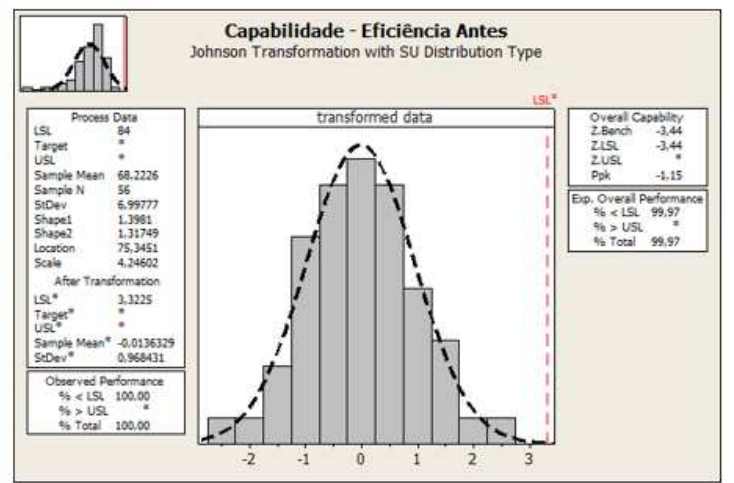

P-value $<0,005$ - Não normal

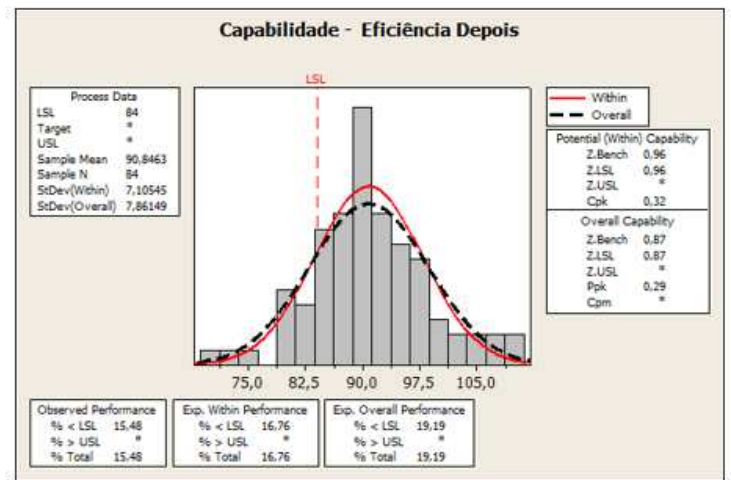

P-value $>0,005$ - Normal

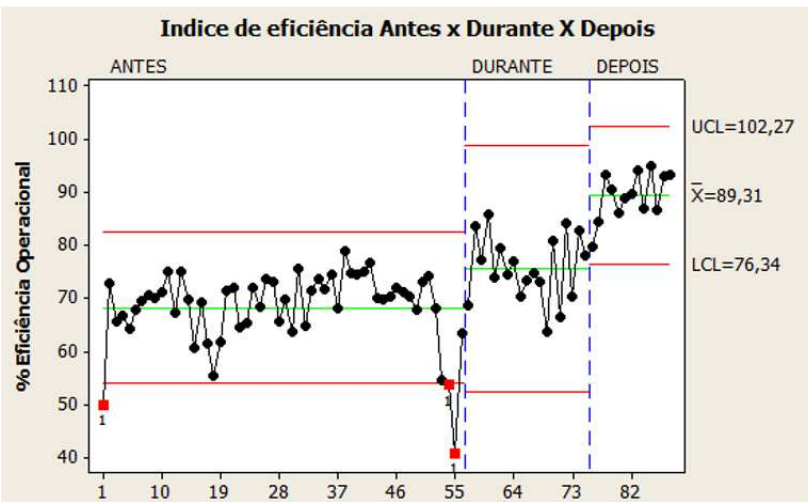

Figura 5: Resultado de capabilidade e \% de eficiência do setor do laminador a frio 


\subsection{Tela EVG}

No setor de telas, para estabilizar a velocidade do equipamento na máxima permitida por tipo de material, foi desenvolvida uma lógica de automação em linguagem STL no CLP S7400 da Siemens, permitindo comandos e informando status na nova IHM OP277 Siemens (plataforma WinccFlexible). O sistema possibilita ajustes de velocidade na função manual porém esse recurso é temporizado, onde o mesmo após zerado toma a decisão de acelerar o equipamento até a velocidade de "set point". Após as implementações nesse equipamento, houve um aumento de performance de OEE de 6,1\%.
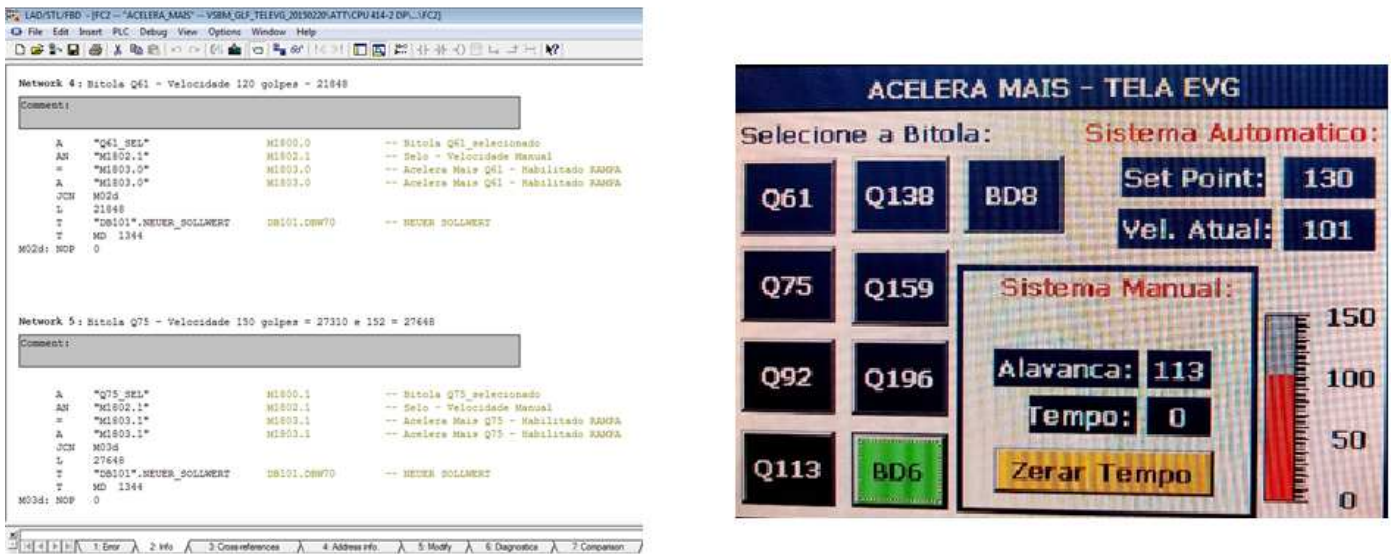

Figura 6: Sistema de velocidade implementado na máquina de Telas

\subsection{Treliça EVG}

No setor de treliça, foi desenvolvida uma nova lógica de automação que permitiu um aumento de velocidade máxima em até $12 \%$. Para garantir a alta performance, foi necessário aumentar a referência em $(\mathrm{Hz})$ dos inversores de frequência Lenze dos motores do loop (Banzo e zig-zag), permitindo um maior giro em RPM, além de otimizar as etapas de sincronismo na lógica entre o cabeçote de soldagem e loop no CLP S7300 (Siemens). Todos os pontos críticos do processo foram inseridos na carta de controle, garantindo a performance dentro das especificações técnica de máquina (corrente, temperatura, torque, velocidade). Após as implementações nesse equipamento, houve um aumento de performance de OEE de $7,1 \%$.

\section{CONCLUSÃo}

Após as análises, ações, resultados apresentados e conhecer que numa área de acabamento siderúrgico existem diversos equipamentos independentes e descentralizados, é possível concluir que um sistema de Aumento de produtividade nos equipamentos através da automatização e otimização dos sistemas de velocidade otimiza "gaps", facilita a gestão operacional e consegue através da tecnologia embarcada em CLPS e interface homem máquina, trazer resultados financeiros e operacionais muito significativos dentro da siderurgia, de forma que a empresa se torne mais competitiva no quadrante operacional e consequentemente no mercado, com custo de aquisição próximo a zero. Após a implementação foi capturado um aumento de $6,62 \%$ de OEE e $5,5 \%$ de produtividade total anual no setor sem custo de aquisição. 


\section{BIBLIOGRAFIA}

1 GARCIA, Claudio. Modelagem e simulação de processos industrias e de sistemas eletromecânicos. São Paulo: Editora da Universidade de São Paulo, 2005.

2 GACS, Grupo de automação e controle de sistemas. Sistemas Eletromecânicos. Disponível em:

<http://www.feng.pucrs.br/ gacs/new/disciplinas/model/apostilas/Aula5.pdf>. Acesso em: 23 abr. 2014.

3 IME, Instituto de matemática e estatítica. Modelagem matemática de sistemas eletromecânicos. Disponível

em:<http://www.ime.usp.br/ oda/contents/01Matem\%E1tica/01Sistemas\%20Din\%E2mi cos/09_Mod_Mat_Sist_Eletromec.pdf>. Acesso em: 23 abr. 2014.

4 OGATA, Katsuhiko. Engenharia de controle moderno. 5. ed. São Paulo: Pearson Prentice Hall, 2010.

5 PINHEIRO, Carlos Alberto Murari; SOUZA, Antonio Carlos de. Introdução à modelagem, análise e simulação de sistemas dinâmicos. Rio de Janeiro: Interciência, 2008.

6 NORMAN, S. NISE, Engenharia de Sistemas de controle. 6.ed.. LTC

7 PINHEIRO, Antonio Carlos Zambroni, Paulo César Rosa, Isaías Lima. Sistemas de controle: Projetos, Simulações e Experiências de Laboratório: Esetec, 2012. 